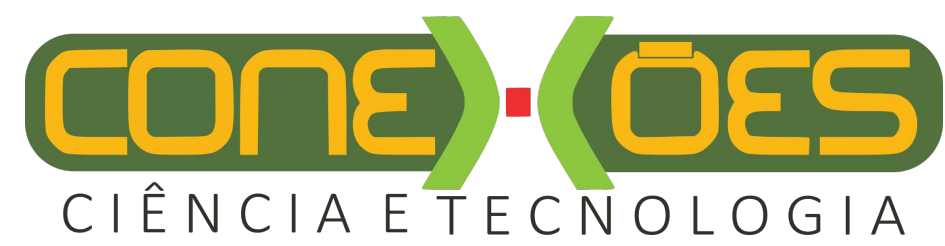

\title{
A COSMOLOGIA QUÂNTICA DE WHEELER-DeWITT E $O$ UNIVERSO DESPEDAÇADO
}

\author{
CELIO RODRIGUES MUNIZ ${ }^{1}$, MARCONY SILVA CUNHA ${ }^{2}$, VALDIR BARBOSA BEZERRA ${ }^{3}$, \\ HORÁCIO SANTANA VIEIRA ${ }^{4}$ \\ ${ }^{1,2}$ Universidade Estadual do Ceará (UECE) \\ ${ }^{3}$ Universidade Federal da Paraíba (UFPB) \\ ${ }^{4}$ Universidade Federal do Rio Grande do Norte (UFRN) \\ <celiomuniz131@gmail.com>,<marcony.cunha@uece.br>,<valdir@fisica.ufpb.br>, \\ $<$ horacio.santana.vieira@hotmail.com>
}

DOI: 10.21439/conexoes.v10ix.999

\begin{abstract}
Resumo. Neste trabalho investigamos, inicialmente, como uma forma de energia escura chamada de phantom, teoricamente responsável por um estado de superaceleração da expansão cósmica, influencia a função de onda do Universo. Isso será feito por meio da obtenção das soluções da equação que descreve o comportamento quântico deste último - a equação de Wheeler-DeWitt - com um termo de ambiguidade no ordenamento dos operadores conjugados associados ao fator de escala cósmico, $a$, e que depende de um parâmetro adicional, $q$. A energia de phantom é implementada no potencial efetivo que surge nesta equação, e o Universo é considerado plano. O primeiro resultado encontrado a partir dessas soluções, para $q=0$, indica que o Universo apresenta uma máxima probabilidade de iniciar sua existência com um tamanho (fator de escala) bem definido, probabilidade tanto maior quanto maior o conteúdo de energia phantom, sendo portanto improvável apresentar uma singularidade inicial do tipo $a=0$. Na abordagem semiclássica adotada aqui, estudamos também como o fator de escala evolui temporalmente, via equação de Hamilton-Jacobi. Mostramos que a singularidade de Big Rip (Grande Despedaçamento) emerge das soluções encontradas, um cenário dramático representado pelo fato de o Universo atingir um fator de escala infinito em um tempo cosmológico finito. Em seguida, avaliamos o que acontece quando, no lugar da energia phantom, incluímos energia escura sob a forma de uma constante cosmológica positiva. Neste caso, mostramos que o Universo não alcança a singularidade de Big Rip, o que fica evidente quando comparamos graficamente o tempo cosmológico em função do fator de escala com o caso anterior.
\end{abstract}

Palavras-chaves: Equação de Wheeler-DeWitt. Energia escura. Big Rip.

\begin{abstract}
In this work, we firstly investigate how a form of dark energy called it phantom, theoretically responsible for a state of superacceleration of cosmic expansion, influences the wave function of the Universe. This will be done by obtaining solutions from the equation describing the Universe quantum behavior - the Wheeler-DeWitt equation - with a term of ambiguity in the ordering of the conjugate operators associated with the cosmic scale factor, $a$, and that depends on an additional parameter, $q$. The phantom energy is implemented in the effective potential that emerges in this equation, and the Universe is considered flat. The first result found from these solutions, for $q=0$, indicates that the Universe presents a maximum probability of starting its existence with a well-defined size (scale factor), probability greater the higher the energy content it phantom, and it is therefore unlikely to present an initial singularity of type $a=0$. In the semiclassical approach adopted here, we also study how the scale factor evolves temporarily, using the Hamilton-Jacobi equation. We show that the Big Rip singularity emerges from the solutions found, a dramatic scenario represented by the fact that the Universe reaches an infinite scale factor in a finite cosmological time. Next, we evaluate what happens when, instead of the energy it phantom, we include dark energy in the form of a positive cosmological constant. In this case, we show that the Universe does not reach the singularity of the Big Rip, which becomes evident when we compare graphically the cosmological time as a function of the scale factor with the previous case.
\end{abstract}

Keywords: Wheeler-DeWitt equation. Dark energy. Big Rip. 


\section{COREE). (DES}

\section{INTRODUÇÃO}

Quando Einstein estendeu as suas equações de campo da Relatividade Geral (RG) de modo a incluir o termo que envolve a constante cosmológica, procurando evitar assim o colapso do Universo perante a ação sempre atrativa da gravidade, não poderia prever que essa ideia, considerada por ele o maior erro de sua vida acadêmica (HARVEY; SCHUCKING, 2000), levaria a um cenário de instabilidade cósmica muito mais dramática do que aquela que ele imaginou, e possivelmente real.

Na verdade, a constante cosmológica, designada por $\Lambda$, está associada a uma forma de energia que poderia ser a responsável final pela expansão acelerada do Universo. A observação desta última, ocorrida em 1998 a partir do brilho de uma supernova (DE LIMA, 2004), é um dos principais marcos da Astrofísica e da Cosmologia desde a primeira comprovação da RG, ocorrida há 100 anos no luminoso céu de Sobral, e a descoberta, pelo astrônomo Edwin Hubble em 1929, de que o Universo estava em expansão, a partir da observação do desvio para o vermelho no espectro eletromagnético da luz emitida por galáxias situadas fora do nosso grupo local (HUBBLE, 1929).

A constante $\Lambda$ (positiva) pode ser associada a um tipo de energia que age repulsivamente em escala cósmica e que é de natureza completamente desconhecida pelos cosmólogos, por isso mesmo chamada de energia escura. Parece ser, atualmente, a forma de energia a predominar largamente sobre os demais tipos presentes no Universo (como radiação eletromagnética, neutrinos e matéria bariônica). Com efeito, físicos e cosmólogos da atualidade travam um vigoroso debate em torno da possibilidade de que essa energia escura seja de fato proveniente das flutuações quânticas do vácuo associado aos diversos campos existentes na natureza (SOLÀ, 2013). O problema é que tal hipótese leva ao que é conhecido como a maior discrepância da história da Física, entre teoria ( $\Lambda$ como resultado das flutuações quânticas do vácuo) e experimento ( $\Lambda$ observado), de cerca de 120 ordens de grandeza (GREENE, 2011).

Entretanto, $\Lambda$ define um limite demarcatório entre outros tipos possíveis de energia escura. Há um parâmetro cosmológico que caracteriza as diversas energias que existem no Universo (ou que podem existir) na forma simples de um fluido perfeito. Esse parâmetro surge na equação de estado que relaciona a pressão do fluido, $p$, e a sua densidade, $\rho$, numa simples relação direta de proporcionalidade: $p=\omega \rho$. Na maior parte dos modelos cosmológicos, o mencionado parâmetro de estado, $\omega$, é uma constante, e quando ele é menor do que zero o modelo indica que a pressão é negativa (pois a densidade de energia é suposta ser positiva), o que res- ponderia por uma expansão acelerada do Universo se tal forma de energia for predominante. A bem da verdade, a expansão acelerada observada desde 1998 é compatível apenas com $-1 / 3>\omega>-1$. Tal intervalo para $\omega$ caracterizaria a chamada quintessência, que, diferentemente da energia escura proveniente de $\Lambda$, teria densidade de energia variável no tempo e no espaço, e que por isso mesmo poderia exercer efeitos locais em escalas astronômicas (BEZERRA et al., 2017), tornando-se todavia menos rarefeita com a expansão do Cosmos em comparação com a matéria e a radiação ordinárias. No limite para $\omega=-1$, a densidade não varia com essa expansão, denotando assim a energia escura associada $\operatorname{com} \Lambda$.

O drama cósmico começaria com a possibilidade da energia escura ter $\omega$ menor que -1 . Neste caso, a densidade de energia cresceria com a expansão do Universo, provocando uma aceleração cada vez maior da expansão, até o Universo atingir um tamanho infinito em um tempo cosmológico finito. Isso teria implicações trágicas, pois o horizonte cosmológico diminuiria paulatinamente até reduzir-se a um ponto. Nesse processo, todas as galáxias, e mesmo as estrelas da nossa Via Láctea, sairiam progressivamente do nosso campo de observação (e sem haver qualquer outra forma de acesso), como também seriam despedaçadas, devido ao caráter extremamente repulsivo dessa forma de energia escura, sinistramente denominada phantom (CALDWELL; KAMIONKOWSKI; WEINBERG, 2003), com o terrível cenário final aqui delineado sendo sugestivamente chamado de Big Rip, ou Grande Despedaçamento.

As observações cosmológicas atuais impõem um limite inferior para $\omega$ da ordem de $-3 / 2$. No presente artigo, trabalharemos com a energia escura associada a $\omega=-5 / 3$, o que nos leva a soluções mais simples para a equação de Wheeler-DeWitt (WdW), que é a equação que descreve a função de onda do Universo. Este seria quântico mesmo em larga escala (NORBURY, 1998), já que não existe um meio ambiente externo a ele por meio do qual seu estado quântico viesse a sofrer decoerência, tornando-se assim um sistema clássico, como os macro-objetos que compõem o nosso mundo (YUROV; YUROV, 2019). Na abordagem semiclássica adotada aqui, combinaremos o estudo da equação $\mathrm{WdW}$ com a de Hamilton-Jacobi, para determinar como o fator de escala do Universo evolui com o tempo, uma vez que a primeira dessas equações não pressupõe nenhuma evolução temporal. Será considerado também na equação de WdW o termo adicional de ambiguidade no ordenamento dos operadores conjugados relativos ao fator de escala. Mostraremos que, de fato, o cenário de Big Rip emerge das soluções encontradas. Em seguida, avalia- 


\section{CORE) (DES \\ CI ÊNCIAE TECNOLOGIA}

\section{A COSMOLOGIA QUÂNTICA DE WHEELER-DeWITT E O UNIVERSO DESPEDAÇADO}

remos o que acontece quando, no lugar da energia phantom, incluímos energia escura advinda da constante cosmológica. Neste caso, mostraremos que o Universo não alcança a singularidade de Big Rip

$\mathrm{O}$ artigo está estruturado como segue: Na seção 2, é feito o estudo das equações de WdW e de HamiltonJacobi, com o objetivo de estudar a dinâmica do Universo para as energias escuras alusivas ao phantom e à constante cosmológica. Na seção 3, são discutidos os resultados obtidos. E, finalmente, na seção 4, são apresentadas as conclusões e perspectivas futuras.

\section{O UNIVERSO QUÂNTICO: A EQUAÇÃO DE WHEELER-DEWITT}

A equação de Wheeler-DeWitt (WdW) na aproximação de mini-superespaço, considerando a métrica de Friedmann-Robertson-Walker (FRW), descreve o comportamento da função de onda do Universo de uma forma muito semelhante à equação de Schrödinger em uma dimensão, com a diferença principal de que aquela não leva em conta a evolução temporal, isto é, não possui derivada em relação ao tempo. No cenário analisado aqui, a equação WdW é escrita como

$$
\begin{aligned}
\left\{\frac{d^{2}}{d a^{2}}+\frac{q}{a} \frac{d}{d a}\right. & -\frac{9 \pi c^{4} a^{2}}{4 \hbar^{2} G^{2}}\left[k c^{2}-\frac{8 \pi G a^{2}}{3 c^{2}} \times\right. \\
& \left.\left.\left.\times \sum_{i} \rho_{\omega_{i}}+\rho_{v}\right)\right]\right\} \Psi(a)=0,
\end{aligned}
$$

onde a função de onda do Universo depende tão somente do seu fator de escala $a$. O termo que envolve o parâmetro adimensional $q$ é oriundo da ambiguidade do ordenamento dos operadores de momento conjugado de $a, \hat{p}_{a}=-i \hbar \partial / \partial a$, e o próprio $a$ na construção do hamiltoniano, uma vez que o fator $a$ desempenha aqui o papel de uma coordenada generalizada. A constante $k$ representa a curvatura do Universo: $k=0$ corresponde ao Universo sem curvatura, plano; $k=1$, com curvatura positiva, fechado sobre si mesmo (Universo de Einstein) e $k=-1$, com curvatura negativa, aberto.

Nesta seção, não será levada em conta a densidade de energia do vácuo, usualmente caracterizada pela constante cosmológica, $\Lambda$, de modo que $\rho_{v}=0$. As demais formas de energia, cujas densidades estão contempladas nos termos $\rho_{\omega_{i}}$, podem ser expressas como (VIEIRA; BEZERRA, 2016).

$$
\rho_{\omega_{i}}=\frac{A_{\omega_{i}}}{a^{3\left(\omega_{i}+1\right)}},
$$

onde os $\omega_{i}$ 's são os parâmetros de estado, que garantem a proporcionalidade entre a pressão e a densidade de energia para cada forma de energia, considerados aqui como fluidos perfeitos.

Consideraremos, inicialmente, apenas a forma de energia phantom, $\operatorname{com} \omega=-5 / 3<-1$. Dessa forma, a equação $\mathrm{WdW}$ se torna

$$
-\frac{\partial^{2} \Psi(a)}{\partial a^{2}}-\frac{q}{a} \frac{\partial \Psi(a)}{\partial a}+V_{e f f}(a) \Psi(a)=0,
$$

onde o potencial efetivo, $V_{e f f}(a)$, é dado por

$$
V_{\text {eff }}(a)=A a^{2}-B a^{6}
$$

na qual os coeficientes $A$ e $B$ são escritos como

$$
A=\frac{9 \pi c^{6} k}{4 \hbar^{2} G^{2}}
$$

$\mathrm{e}$

$$
B=\frac{6 \pi^{2} c^{2}}{\hbar^{2} G} A_{p}
$$

$\operatorname{com} A_{p}$ sendo uma constante proporcional à densidade de energia phantom em algum instante da história do Universo. Segundo nosso modelo, esta forma de energia será predominante no Universo, principalmente em um tempo cosmológico futuro (distante?), o qual se supõe também dotado de curvatura nula $(k=0)$, o que corresponde às mais recentes observações do satélite Planck (AGHANIN et al., 2018).

Para encontrarmos a solução de (3), vamos proceder à transformação na variável dependente, tal que $\Psi(a)=$ $a^{(1-q) / 2} \phi(a)$, de modo que ficamos com a equação

$$
a^{2} \phi^{\prime \prime}(a)+a \phi^{\prime}(a)+\left[B a^{8}-\left(\frac{q-1}{2}\right)^{2}\right] \phi(a)=0 \text {. }
$$

Operando-se agora a transformação na variável independente na forma $z=\sqrt{B} a^{4} / 4$, temos

$$
z^{2} \phi^{\prime \prime}(z)+z \phi^{\prime}(z)+\left[z^{2}-\left(\frac{q-1}{8}\right)^{2}\right] \phi(z)=0 .
$$

A expressão acima tem a forma da equação de Bessel, a saber, $z^{2} Y^{\prime \prime}(z)+z Y^{\prime}(z)+\left(z^{2}-\nu^{2}\right) Y(z)=0$, cuja solução é dada em termos das funções de Bessel de primeira e segunda espécies, $J_{\nu}(z)$ e $Y_{\nu}(z)$, respectivamente. Mas podemos transformar a segunda de modo a deixar a solução apenas em função de $J_{\nu}(z)$, o que nos será bastante útil. Assim, após voltarmos para as variáveis dependente e independente originais, a solução para (7) será dada por

$$
\begin{aligned}
& \left.\Psi(a)=C_{1} B^{\frac{1-q}{16}} \Gamma\left(\frac{9-q}{8}\right) a^{\frac{1-q}{2}} J_{\frac{1-q}{8}} \frac{\sqrt{B} a^{4}}{4}\right) \\
& \left.+C_{2} B^{\frac{1-q}{16}} \Gamma\left(\frac{7+q}{8}\right) a^{\frac{1-q}{2}} J_{\frac{q-1}{8}} \frac{\sqrt{B} a^{4}}{4}\right),
\end{aligned}
$$




\section{CORE) (DES \\ CI ÊNCIAETECNOLOGIA}

\section{A COSMOLOGIA QUÂNTICA DE WHEELER-DeWITT E O UNIVERSO DESPEDAÇADO}

onde $C_{1}$ e $C_{2}$ são as constantes de normalização.

Nas seções seguintes, ao analisarmos a dinâmica do Universo e o cenário de Big Rip, trabalharemos com dois diferentes valores inteiros do parâmetro $q$ de modo a selecionar as soluções linearmente independentes (L.I.) da Eq. (9), a saber: $q=0$, que permite a escolha da primeira delas, uma vez que a segunda solução dá valores divergentes para a função de onda na origem, dado que o índice de $J_{\nu}(z)$ fica um não inteiro negativo. Esse valor para $q$ também não leva a divergências no infinito. A outra escolha é $q=1$, que seleciona as duas soluções ao mesmo tempo, sem divergências na origem nem no infinito. Notemos que não há nenhum valor para $q$ que selecione apenas a segunda das funções L.I., sem que haja complicações envolvendo divergências. Fazemos notar também que $q=0$ fornece a condição de contorno de Hartle-Hawking, segundo a qual a função de onda do Universo se anula na origem. Já a condição de contorno de Linde, em que a função de onda é finita na origem (VILENKIN, 1999), ocorre apenas para $q=1$.

Assim, para $q=0, C_{2}=0$, e integrando-se o quadrado da primeira solução L.I., dada na Eq. (9), de $a=0$ a $a=\infty$, a constante de normalização $C_{1}$ fica dada por

$$
C_{1}=\frac{\sqrt[4]{2} \sqrt[16]{B} \Gamma\left(\frac{7}{8}\right)}{\Gamma\left(\frac{9}{8}\right) \sqrt{\Gamma\left(\frac{5}{4}\right)}},
$$

e $C_{2}=0$, de modo que a função de onda do Universo é exatamente

$$
\Psi(a)=\frac{\sqrt{a} \sqrt[8]{B} \Gamma\left(\frac{7}{8}\right) J_{\frac{1}{8}}\left(\frac{\sqrt{B} a^{4}}{4}\right)}{\sqrt[8]{2} \sqrt{\Gamma(5 / 4)}} .
$$

Plotamos, a título de completeza, no gráfico da Fig. (1), o quadrado de $\Psi$ em função de $a$ e de $B$. É interes-

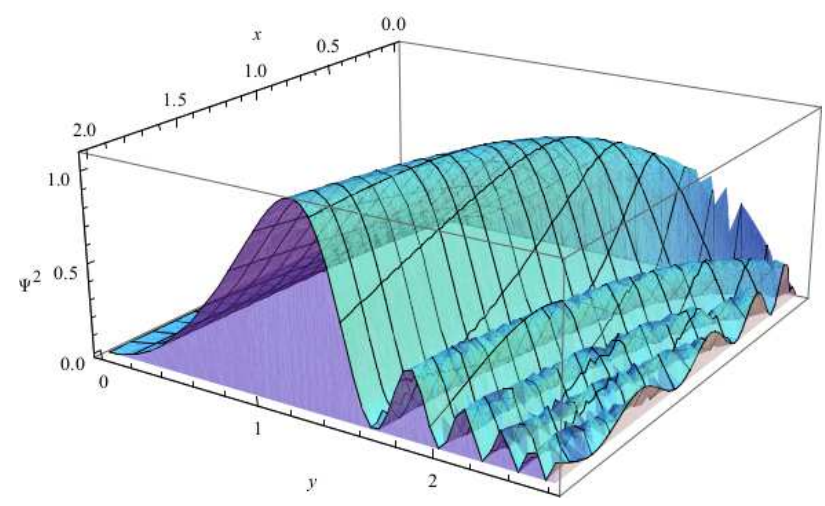

Figura 1: O quadrado da função de onda, $\Psi^{2}$, em função de $B$ (eixo $x$ ) e de $a$ (eixo $y$ ), para $q=0$. sante observarmos que o Universo apresenta uma probabilidade bastante alta de iniciar sua existência com um tamanho (fator de escala) bem definido, probabilidade que será tanto mais alta quanto maior for o conteúdo de energia phantom (proporcional a $B$ ), e que a singularidade inicial tipo $a=0$, portanto, é improvável.

\section{A DINÂMICA DO UNIVERSO}

Esta seção seguirá de perto o método descrito em (VIEIRA, BEZERRA, 2016), o qual recorre a uma abordagem semiclássica através do estudo da equação de Hamilton-Jacobi, já que não se pode descrever a dinâmica do Universo simplesmente por meio da equação (quântica) de Wheeler-deWitt, que sabemos não descrever a evolução temporal do Universo. Assim, como a função de onda $\Psi(a)$ depende apenas de uma variável, $a$, que é o fator de escala do Universo, ela pode ser reescrita como

$$
\Psi(a)=R(a) \mathrm{e}^{i S(a)},
$$

onde $R$ e $S$ são funções reais do fator de escala, esta última sendo a ação do modelo. Segue que o módulo quadrado da função de onda do Universo pode ser escrito como

$$
|\Psi(a)|^{2}=R^{2}(a) .
$$

Da Mecânica Quântica, temos que a densidade de corrente de probabilidade é dada por

$$
j^{a} \propto\left[\Psi^{*}\left(\partial_{a} \Psi\right)-\Psi\left(\partial_{a} \Psi^{*}\right)\right],
$$

garantindo a lei de conservação

$$
\partial_{a} j^{a}=0
$$

Substituindo a Eq. (12) na Eq. (14), obtemos

$$
j^{a}=-\hbar R^{2} \frac{\partial S}{\partial a} .
$$

Integrando a Eq. (15), temos

$$
j^{a}=C_{0},
$$

onde $C_{0}$ é uma constante arbitrária. Assim, das Eqs. (16) e (17), concluímos que

$$
-\hbar R^{2} \frac{\partial S}{\partial a}=C_{0}
$$

Agora, podemos usar o formalismo de HamiltonJacobi da Mecânica Quântica para escrever a seguinte relação entre a ação e o momento canônico:

$$
p_{a}=\frac{\partial S}{\partial a}=\frac{\partial L}{\partial \dot{a}}=-\frac{3 \pi c^{2}}{2 G} \dot{a} a
$$




\section{CORE) (DES \\ CIÊNCIA E TECNOLOGIA}

\section{A COSMOLOGIA QUÂNTICA DE WHEELER-DeWITT E O UNIVERSO DESPEDAÇADO}

onde $L$ é o lagrangiano do Universo de FriedmannRobertson-Walker, conforme (VIEIRA, BEZERRA, 2016). Assim, das Eqs. (18) e (19), obtemos

$$
R^{2}=C_{0} \frac{2 G}{3 \hbar \pi c^{2} \dot{a} a} .
$$

\subsection{O Grande Despedaçamento}

Da expressão (20), podemos obter a evolução do fator de escala do Universo com o tempo. Considerando primeiramente o valor $q=0$, e usando-se a Eq. (13), integramos esta equação de $\mathrm{t}=\mathrm{t}_{0}$ até um certo $\mathrm{t} \mathrm{e}$ de $\mathrm{a}=0$ até $\mathrm{a}=\infty$, obtendo

$$
t=t_{0}+C_{3} \frac{\hbar c^{2}}{G B^{1 / 4}},
$$

com $C_{3}$ sendo uma nova constante. Por outro lado, tomando-se o valor $q=1$, que seleciona a solução geral (9) para a função de onda do Universo, sem gerar divergências, podemos substituir esta em (20) e integrá-la novamente de $a=0$ a $a=\infty$. Encontramos mais uma vez um valor finito para $\left(t-t_{0}\right), \operatorname{com} C_{3}$ sendo diferente do caso anterior, e as demais constantes, idênticas.

Ambas as soluções para o tempo cosmológico em função do fator de escala, considerando-se $q=0$ e $q=$ 1 , resultaram, portanto, em um Universo expandindo-se ao infinito em um tempo de existência finito. Esse cenário verdadeiramente estranho e perturbador é denominado Big Rip, que traduzimos aqui como o Grande Despedaçamento. Note da Eq. (21)) que quando a energia de phantom tende a zero, a singularidade de Big Rip tende a desaperecer, pois o tempo cosmoló-gico vai ao infinito com o crescimento sem limites do fator de escala.

\subsection{Um destino cósmico mais feliz}

Analisaremos agora a situação em que a densidade de energia do Universo está associada exclusivamente ao vácuo, $\rho_{v}$, na forma de uma constante cosmológica positiva, $\Lambda$. Neste caso, a densidade de energia mantém-se a mesma, enquanto o Universo se expande, e o potencial efetivo, considerando-se ainda $k=0$, será $V_{\text {eff }}(a)=-B_{\Lambda} a^{4}$, onde $B_{\Lambda}=\frac{3 \pi c^{6}}{4 \hbar^{2} G^{2}} \Lambda$. A solução da equação (3) pode ser dada mais uma vez em termos da função de Bessel $J_{\nu}(z)$, na forma

$$
\begin{aligned}
& \Psi(a)=C_{1} B_{\Lambda}^{\frac{1-q}{12}} a^{\frac{1-q}{2}} \Gamma\left(\frac{7-q}{6}\right) J_{\frac{1-q}{6}}\left(\frac{\sqrt{B_{\Lambda}} a^{3}}{3}\right) \\
& +C_{2} B_{\Lambda}^{\frac{1-q}{12}} a^{\frac{1-q}{2}} \Gamma\left(\frac{5+q}{6}\right) J_{\frac{q-1}{6}}\left(\frac{\sqrt{B_{\Lambda}} a^{3}}{3}\right)
\end{aligned}
$$

Tomando-se o valor $q=0$ e a primeira das soluções L.I. acima, pelas razões expostas anteriormente e que são válidas aqui também, podemos fazer a substituição na equação (20), integrando-a mais uma vez em $a$, no intervalo $[0, \infty)$, e no tempo, de $t_{0}$ a $t$. Verificamos, como era de se esperar, que o valor encontrado para $t-t_{0}$ diverge, significando que o Universo atinge o tamanho infinito em um tempo também infinito. Podemos igualmente mostrar que o resultado diverge também para $q=1$. Um destino menos trágico para o Universo, portanto.

\section{RESULTADOS E DISCUSSÃO}

O gráfico da Fig. (2), que representa o tempo cosmológico $t$ (com $\left.t_{0}=0\right)$, em função do fator de escala $(a)$, ilustra bem o que acontece com a expansão do Universo governado unicamente pela energia phantom. Para o caso de $q=0$, ele inicia sua existência com uma expansão bastante acentuada, mais que no caso com $q=1$, o que se pode depreender mais facilmente da Fig. (3), expansão que em seguida se atenua, passando a crescer com relativa lentidão comparando-se com este último caso. Não podemos afirmar, entretanto, que a rápida expansão registrada até o "cotovelo" da curva (no início do Universo, portanto) corresponde à Inflação Cósmica, pois não incluímos nenhum outro ingrediente em nosso modelo, como o campo de inflaton e outras formas de energia, com certeza presentes no Universo primordial.

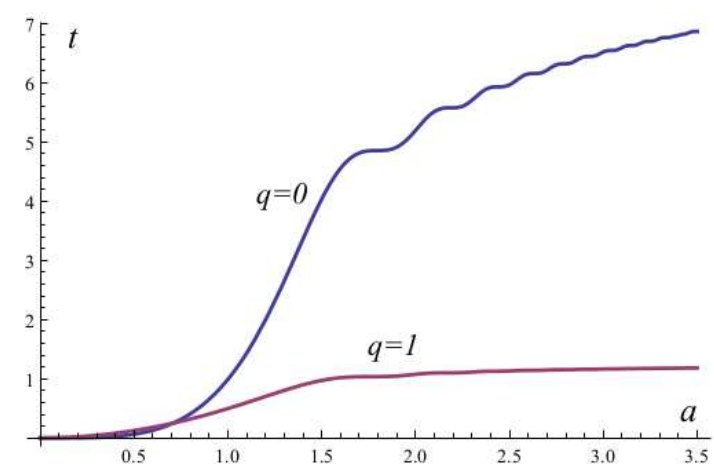

Figura 2: Tempo cosmológico, $t$, em função do fator de escala, $a$, para $q=0$ e $q=1$. Todas as demais constantes foram igualadas à unidade.

Ainda segundo o gráfico apresentado na Fig. (2), em determinado momento da história cósmica quando o conteúdo de energia phantom predomina sobre os demais, ocorre uma sucessão de acelerações e desacelerações na expansão do Universo, representada pelas ondulações que a curva obedece, bem visíveis no caso com $q=0$. Todavia, a amplitude dessas oscilações vai diminuindo enquanto o Universo expande-se cada vez mais rápido, até este atingir um tamanho infinito em um tempo finito de existência, dado pela equação 


\section{CORE) (DES \\ CIÊNCIA E TECNOLOGIA}

A COSMOLOGIA QUÂNTICA DE WHEELER-DeWITT E O UNIVERSO DESPEDAÇADO

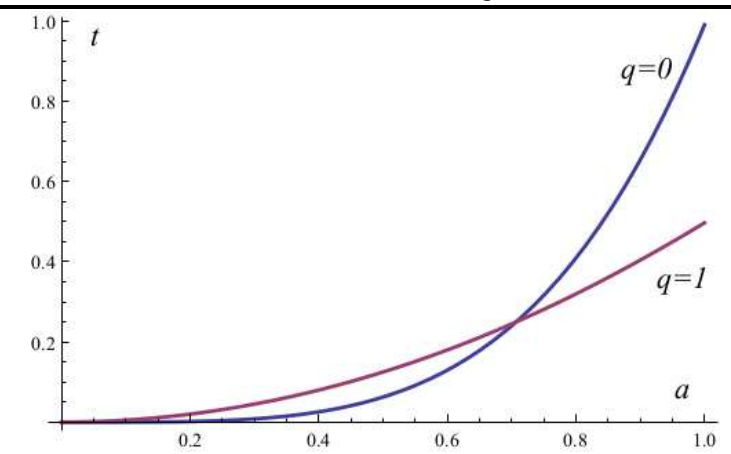

Figura 3: Detalhe do gráfico anterior, para os tempos iniciais de vida do Universo, $\operatorname{com} q=0$ e $q=1$. Todas as demais constantes foram igualadas à unidade.

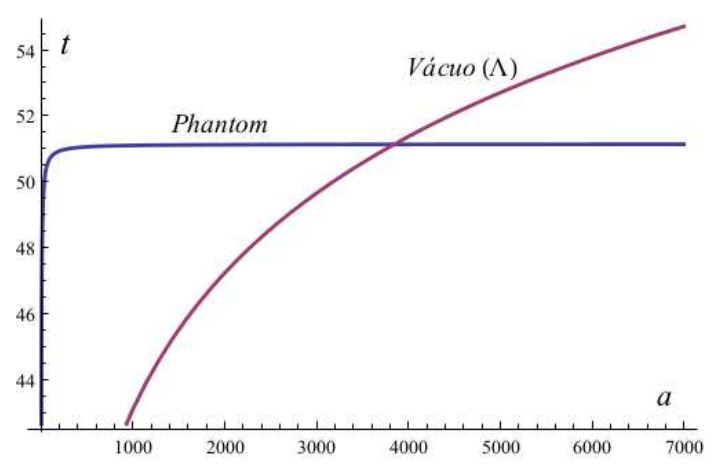

Figura 4: Comparação entre os tempos cosmológicos, em função do fator de escala, para os Universos com predomínio de energia phantom e de energia do vácuo, esta na forma de uma constante cosmológica (positiva). Todas as constantes foram igualadas à unidade.

(21). Desta expressão, percebe-se que quanto maior o conteúdo de energia phantom, menor será o tempo para o Universo chegar a esse estado, chamado de Big Rip como já comentado anteriormente, pois presume-se que nada resistirá a essa expansão desenfreada, nem mesmo as partículas elementares, que se desintegrarão perante força repulsiva tão violenta, com o próprio tecido do espaço-tempo tendendo a se romper. Como a Fig. (2) ainda nos revela, o caso $\operatorname{com} q=1$ chega a ser mais dramático, pois o estado de Big Rip é atingido mais rapidamente ainda, mesmo levando-se em conta que ambos os Universos possuem um mesmo valor para o conteúdo de energia phantom inicial, $A_{p}$.

A tabela 1 mostra o que pode ocorrer em um tempo cosmológico futuro com as macro e microestruturas que compõem o Universo. Essa tabela foi extraída de (CALDWELL; KAMIONKOWSKI, 2003), que supôs uma energia de phantom com parâmetro de estado $\omega=-3 / 2$. Para o caso analisado neste trabalho $(\omega=-5 / 3)$, os eventos seriam ainda mais dramáticos, pois o Big Rip ocorreria daqui a cerca de 16 bilhões de
Tabela 1: Tempos cosmológicos dos principais eventos do Universo, do Big Bang ao Big Rip

\begin{tabular}{cc} 
Tempo Cosmológico & Evento \\
\hline $10^{-43} \mathrm{~s}$ & Era de Planck \\
$\sim 10^{-36} \mathrm{~s}$ & Inflação Cósmica \\
até $3 \mathrm{~min}$ & Nucleossíntese dos \\
& elementos leves \\
$10^{5}$ anos & Formação dos átomos \\
$10^{9}$ anos & Primeiras galáxias \\
$1,4 \times 10^{10}$ anos & Hoje \\
$10^{9}$ anos para o Big Rip & Dissolução dos \\
& aglomerados de galáxias \\
$6 \times 10^{7}$ anos para o Big Rip & Dissolução da \\
3 meses para o Big Rip & Via Láctea \\
30 min para o Big Rip & Dissolução do \\
$10^{-19}$ s para o Big Rip & Sistema Solar \\
& Explosão da Terra \\
$3,5 \times 10^{10}$ anos & Dissociação \\
& dos Átomos \\
& Big Rip
\end{tabular}

anos.

\section{CONCLUSÕES}

Neste artigo, analisamos as soluções da equação quântica de Wheeler-DeWitt que descreve a função de onda do Universo na Cosmologia de FRW, com um termo adicional que caracteriza a ambiguidade na multiplicação dos operadores conjugados associados ao fator de escala, $a$. Tal termo depende de um parâmetro, $q$, ao qual foram atribuídos os valores $q=0$ e $q=1$, de tal modo a eliminar divergências na função de onda, tanto na origem como no infinito. Também trabalhamos com o cenário mais provável do Universo com curvatura nula, $k=0$.

Inicialmente, supomos a presença de energia escura com parâmetro de estado $\omega$ menor que -1 , a saber, $\omega=-5 / 3$, denominada phantom, e encontramos, ao analisar a solução da equação de WdW com $q=0$, que o Universo apresenta uma probabilidade bastante alta de iniciar sua existência com um tamanho (fator de escala) bem definido, probabilidade tanto mais alta quanto maior o conteúdo de energia phantom, e que a singularidade inicial tipo $a=0$ é, portanto, improvável.

Em seguida, mostramos através do estudo da dinâmica cósmica, isto é, de como o fator de escala evolui com o tempo por meio da equação de Hamilton-Jacobi, que esse fator tende ao infinito em um tempo cosmológico finito, para ambos os valores $q=0$ e $q=1$, confirmando o que teorias puramente clássicas, baseadas nas soluções da equação de Friedmann, já prediziam. Tal 


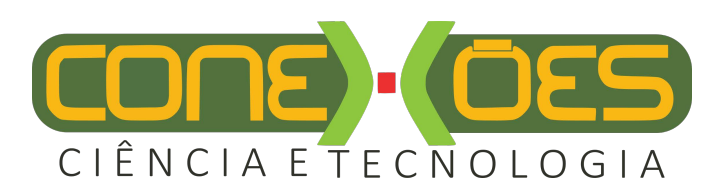

A COSMOLOGIA QUÂNTICA DE WHEELER-DeWITT E O UNIVERSO DESPEDAÇADO

estado singular é designado na literatura por Big Rip, e é geralmente descrito como a culminância do período da história cósmica em que aquela forma de energia passa a predominar sobre todas as outras, período em que todas as estruturas materiais que formam o Universo, não importando o seu grau de coesão, desde aglomerados de galáxias até as partículas subatômicas, serão esfaceladas devido ao caráter extremamente repulsivo que essa energia assume, tornando-se mais densa à medida que o Universo se expande e tornando-se, com isso, cada vez mais influente e destrutiva. Essa singularidade cósmica é prevista ocorrer em poucas dezenas de bilhões de anos a partir do Big Bang.

Também examinamos o caso em que a energia escura está na forma da constante cosmológica, $\Lambda>0$, que corresponde exatamente a $\omega=-1$. Depreendese da análise feita que o cenário de Big Rip não caracteriza o futuro do Universo cuja evolução é governada por essa forma de energia, pois mostramos que o fator de escala tende ao infinito apenas em um tempo cosmológico igualmente infinito. Isso ficou claro quando comparamos por meio do gráfico da Fig. (4) a evolução do fator de escala para as duas formas de energia escura aqui analisadas.

Pretendemos generalizar esse resultado em um outro trabalho, considerando a perspectiva semiclássica que adotamos aqui, para qualquer forma de energia escura em que $\omega<-1$, mostrando que de fato ocorre a singularidade do Big Rip apenas para esses valores do parâmetro de estado, e que esse cenário não ocorre para qualquer $\omega \geq-1$. Esperamos fazer isso num futuro próximo, bem antes do Grande Despedaçamento.

\section{AGRADECIMENTOS}

Os autores agradecem o apoio parcial ao $\mathrm{CNPq}$ e à FUNCAP, esta através do PRONEM PNE-011200085.01.00/16.

\section{REFERÊNCIAS}

AGHANIN, N. et al., Planck 2018 results. VI. Cosmological parameters. Planck Collaboration. Disponível em: <https://arxiv.org/pdf/1807.06209.pdf>.

BEZERRA, V. B.; CUNHA, M. S.; FREITAS, L. F. F.; MUNIZ, C. R.; TAHIM, M. O. Casimir effect in the Kerr spacetime with quintessence, Modern Physics Letters A 32, 01, 1750005 (2017).

CALDWELL, R. R.; KAMIONKOWSKI, M.; Weinberg, N. N. Phantom Energy: Dark Energy with $\omega<-1$ Causes a Cosmic Doomsday, Physical Review

\section{Letters 91, 071301, 2003.}

DE LIMA, J. A. S. Cosmologia, quintessência e aceleração do universo, REVISTA USP, São Paulo, n.62, 134, 2004.

GREENE, B. A Realidade Oculta: Universos Paralelos $e$ as Leis Profundas do Cosmo. São Paulo: Cia das Letras, 2011.

HARVEY, A.; SCHUCKING, E. Einstein's mistake and the cosmological constant. American Journal of Physics 68, 8, 723, 2000.

HUBBLE, E., A relation between distance and radial velocity among extra-galactic nebulae, PNAS 15, 3, 168, 1929.

NORBURY, J. W. From Newton's laws to the WheelerDeWitt equation, European Journal of Physics 19, 2, 1998.

SOLÀ, J., Cosmological constant and vacuum energy: old and new ideas, J. Phys.: Conf. Ser. 453, 012015, 2013.

VIEIRA, H. S.; BEZERRA, V. B. Class of solutions of the Wheeler-DeWitt equation in the FriedmannRobertson-Walker universe, Physical Review D 94, 023511 (2016).

VILENKIN, A. The quantum cosmology debate. AIP Conference Proceedings 478, 23 (1999).

YUROV, A.; YUROV, V. The Day the Universes Interacted: Quantum Cosmology without a Wave function. 2019. Disponível em: <https://arxiv.org/pdf/ 1901.01873.pdf>. 